Rev. Elev. Méd. vét. Pays trop., 1971, 24 (1): 37-41

\title{
A propos de l'action du Bitin-S et du Bromophénophos sur les formes immatures de Fasciola gigantica
}

\author{
par M. GRABER, E. BIRGI et P. M. TRONCY (*)
}

\begin{abstract}
RESUME
En pays tropical, le traitement des distomatoses récentes dues à des formes immatures de Fasciola gigantica, en migration dans le parenchyme hépatique, est difficile.

L'Acédist, chez le zébu, est inutilisable, car la dose habituelle $(10-12,5 \mathrm{mg} / \mathrm{kg})$ doit être plus que doublée et, dans ces conditions, le médicament risque de devenir dangereux pour la santé de l'animal.

Chez le mouton, le Bitin-S, quoique très efficace vers $40 \mathrm{mg} / \mathrm{kg}$, ne devra pas être administré à des ovins en trop mauvais état.

Par contre, chez le zébu, le médicament est, en général, bien toléré. Pour des infestations de 8 à 13 semaines, la dose préconisée est de $40 \mathrm{mg} / \mathrm{kg}$, avec un pourcentage d'efficacité supérieur à 90 p. 100. Celles de moins de 8 semaines exigent une posologie un peu plus élevée.
\end{abstract}

Le Bis (2-Hydroxy 3,5 Dichlorophényl) Sulfoxyde ( ${ }^{1}$ ) est un médicament dont le pouvoir antidistomien est connu depuis les travaux d'ODA (1962) et d'UENO et Collab. (1964 a et b) au Japon, de GUILHON et GRABER (1966 et 1967) en France et en République du Tchad, de CHROUSTOVA et Collab. (1969) en Tchéchoslovaquie et de DELAK et MAGUD (1968) en Pologne. La dose de $30 \mathrm{mg} / \mathrm{kg}$ permet, en général, la destruction et l'élimination des Fasciola adultes et mûres localisées dans les voies biliaires (canaux et vésicule) du mouton et du zébu.

Quant au Bromophénophos ('), c'est un Ester phosphorique tétrabromé mis au point en Hollande par VAN DER MEER et POUWELS

(*) Institut d'Elevage et de Médecine vétérinaire des Pays tropicaux - Laboratoire de Farcha - Fort-Lamy République du Tchad; Service de Parasitologie - Ecole nationale vétérinaire de Lyon.

(1) Bitin-S.A.K.T. 501, Tanabé Pharmaceuticals; en France, Disto - 5 Cogla.

() Acédist I.S.T.
(1969). Chez le mouton, il est actif sur les Distomes (Fasciola hepatica et Fasciola gigantica) à la dose de $16-20 \mathrm{mg} / \mathrm{kg}$ et, sur les immatures de 6-10 semaines, à des doses plus élevées de l'ordre de 22 à $25 \mathrm{mg} / \mathrm{kg}$ (KRUYT et VAN DER STEEN, 1969; GUILHON et GRABER (1970 a et b).

Chez les bovins, les chercheurs néerlandais (REINDEERS, 1969; KOOPMAN, 1969) obtiennent à $12 \mathrm{mg} / \mathrm{kg}$ de bons résultats, quel que soit l'âge des parasites (Fasciola hepatica).

Cependant, les renseignements obtenus en Afrique tropicale sont encore incomplets et un certain nombre de points demandent à être précisés. Ils concernent essentiellement, chez le mouton et chez le zébu, l'efficacité du Bitin-S à l'égard des jeunes Douves en migration dans le parenchyme hépatique et, chez le zébu, celle de l'Acédist. Le Trématode en cause est, dans tous les cas, Fasciola gigantica, espèce très répandue au Sud du Sahara. 
TABLEAU $N^{\circ} I$

Action du Bitin-s sur les formes immatures de Fascicla gigantica du mouton.

\begin{tabular}{|c|c|c|c|c|c|}
\hline \multirow{2}{*}{ Mouton $\mathrm{N}^{\circ}$} & \multirow{2}{*}{$\begin{array}{l}\text { Doses } \\
\text { mg/kg }\end{array}$} & \multirow{2}{*}{$\begin{array}{l}\text { Age des douves } \\
\text { jours }\end{array}$} & \multicolumn{2}{|c|}{$\begin{array}{c}\text { Nombre de parasites retrouvés } \\
\text { à } l^{\prime} \text { autopsie }\end{array}$} & \multirow{2}{*}{ Efficacité } \\
\hline & & & Vivants & Morts & \\
\hline 1 & 40 & 42 & 0 & 5 & Totale \\
\hline $\begin{array}{l}2 \\
3\end{array}$ & $\begin{array}{l}40 \\
60\end{array}$ & $\begin{array}{l}49 \\
49\end{array}$ & $\begin{array}{l}0 \\
0\end{array}$ & $\begin{array}{r}6 \\
13\end{array}$ & $\begin{array}{l}\text { Totale } \\
\text { Totale }\end{array}$ \\
\hline $\begin{array}{l}4 \\
5\end{array}$ & $\begin{array}{l}25 \\
30\end{array}$ & $\begin{array}{l}56 \\
56\end{array}$ & $\begin{array}{l}55 \\
27\end{array}$ & $\begin{array}{l}0 \\
0\end{array}$ & $\begin{array}{l}\text { Nulle } \\
\text { Nulle }\end{array}$ \\
\hline $\begin{array}{l}6 \\
7 \\
8\end{array}$ & $\begin{array}{l}25 \\
30 \\
40\end{array}$ & $\begin{array}{l}75 \\
75 \\
75\end{array}$ & $\begin{array}{r}14 \\
9 \\
0\end{array}$ & $\begin{array}{l}44 \\
37 \\
45\end{array}$ & $\begin{array}{l}75,8 \text { p. } 100 \\
80 \text { p. } 100 \\
\text { Totale }\end{array}$ \\
\hline 9 & 40 & 91 & 1 & 19 & p. 100 \\
\hline
\end{tabular}

TABLEAU N"II

Action du Bitin-s sur les formes immatures de Fasciola gigantica du zébu.

\begin{tabular}{|c|c|c|c|c|c|}
\hline \multirow[t]{2}{*}{ Bouvilion $\mathrm{N}^{\circ}$} & \multirow{2}{*}{$\begin{array}{l}\text { Doses } \\
\mathrm{mg} / \mathrm{kg}\end{array}$} & \multirow{2}{*}{$\begin{array}{c}\text { Age des douves } \\
\text { Jours }\end{array}$} & \multicolumn{2}{|c|}{$\begin{array}{c}\text { Nombre de parasites retrouvés } \\
\text { à l'autopsie }\end{array}$} & \multirow{2}{*}{ Efflcacltê } \\
\hline & & & Vivants & Morts & \\
\hline 1 & 30 & 42 & 42 & 0 & Nulle \\
\hline 2 & $\begin{array}{l}40 \\
60\end{array}$ & $\begin{array}{l}42 \\
42\end{array}$ & $\begin{array}{c}91 \\
\text { (mouvements } \\
\text { ralentis) } \\
0\end{array}$ & $\begin{array}{r}0 \\
84\end{array}$ & $\begin{array}{l}\text { Nulle } \\
\text { Totale }\end{array}$ \\
\hline $\begin{array}{l}4 \\
5 \\
6\end{array}$ & $\begin{array}{l}30 \\
40 \\
60\end{array}$ & $\begin{array}{l}56 \\
56 \\
56\end{array}$ & $\begin{array}{r}57 \\
2 \\
0\end{array}$ & $\begin{array}{l}58 \\
98 \\
93\end{array}$ & $\begin{array}{l}50,4 \text { p. } 100 \\
98 \text { p. } 100 \\
\text { Totale }\end{array}$ \\
\hline $\begin{array}{l}7 \\
8 \\
9\end{array}$ & $\begin{array}{l}30 \\
40 \\
60\end{array}$ & $\begin{array}{l}71 \\
71 \\
71\end{array}$ & $\begin{array}{l}0 \\
0 \\
0\end{array}$ & $\begin{array}{l}144 \\
107 \\
107\end{array}$ & $\begin{array}{l}\text { Totale } \\
\text { Totale } \\
\text { Totale }\end{array}$ \\
\hline $\begin{array}{l}10 \\
11\end{array}$ & $\begin{array}{r}30 \\
.40\end{array}$ & $\begin{array}{l}91 \\
91\end{array}$ & $\begin{array}{l}15 \\
12\end{array}$ & $\begin{array}{l}89 \\
70\end{array}$ & $\begin{array}{l}85,5 \text { p. } 100 \\
85,3 \text { p. } 100\end{array}$ \\
\hline
\end{tabular}

TABLEAU $N^{\bullet}$ III

Action de 1'Acedlat sur le's formes 1mmatures de Fasciola gigantica du zébu.

\begin{tabular}{|c|c|c|c|c|c|}
\hline \multirow{2}{*}{ Bouv111on $\mathrm{N}^{*}$} & \multirow{2}{*}{$\begin{array}{l}\text { Doses } \\
\text { mg } / \mathrm{kg}\end{array}$} & \multirow{2}{*}{$\begin{array}{c}\text { Age des douves } \\
\text { Jours }\end{array}$} & \multicolumn{2}{|c|}{$\begin{array}{c}\text { Nombre de parasites retrouvés } \\
\text { à l'autopsie }\end{array}$} & \multirow{2}{*}{ Efficacitê } \\
\hline & & & Vivants & Morts & \\
\hline$\frac{1}{2}$ & $\begin{array}{l}20 \\
25\end{array}$ & $\begin{array}{l}43 \\
43\end{array}$ & $\begin{array}{l}77 \\
92\end{array}$ & $\begin{array}{l}0 \\
0\end{array}$ & $\begin{array}{l}\text { Nulle } \\
\text { Nulle }\end{array}$ \\
\hline $\begin{array}{l}3 \\
4 \\
5\end{array}$ & $\begin{array}{l}15 \\
20 \\
25\end{array}$ & $\begin{array}{l}55 \\
55 \\
55\end{array}$ & $\begin{array}{r}266 \\
140 \\
83\end{array}$ & $\begin{array}{l}0 \\
2 \\
4\end{array}$ & $\begin{array}{l}\text { Nulle } \\
1,4 \text { p.100 } \\
4,6 \text { p.100 }\end{array}$ \\
\hline $\begin{array}{l}6 \\
7\end{array}$ & $\begin{array}{l}15 \\
20\end{array}$ & $\begin{array}{l}72 \\
72\end{array}$ & $\begin{array}{r}155 \\
63\end{array}$ & $\begin{array}{l}22 \\
34\end{array}$ & $\begin{array}{l}12,3 \text { p. } 100 \\
35 \text { p. } 100\end{array}$ \\
\hline $\begin{array}{l}8 \\
9\end{array}$ & $\begin{array}{l}10 \\
10\end{array}$ & $\begin{array}{l}84 \\
84\end{array}$ & $\begin{array}{r}102 \\
88\end{array}$ & $\begin{array}{l}0 \\
3\end{array}$ & $\begin{array}{l}\text { Nu11e } \\
3,3 \text { p.100 }\end{array}$ \\
\hline $\begin{array}{l}10 \\
11\end{array}$ & $\begin{array}{l}10 \\
10\end{array}$ & $\begin{array}{l}104 \\
103\end{array}$ & $\begin{array}{l}0 \\
0\end{array}$ & $\begin{array}{l}31 \\
18\end{array}$ & $\begin{array}{l}\text { Totale } \\
\text { Totale }\end{array}$ \\
\hline
\end{tabular}




\section{MATERIEL ET METHODE}

Neuf moutons ( ${ }^{3}$ ) de 23 à $36 \mathrm{~kg}$ et vingt-deux bouvillons de 114 à $257 \mathrm{~kg}$ ont été utilisés. Ils ont été achetés dans le nord-ouest du Tchad où la distomatose est peu fréquente. Pour plus de sécurité, les animaux ont été soumis à des séries d'examens coproscopiques qui se sont tous révélés négatifs.

Passé ce temps, les ovins ont reçu chacun 150 métacercaires de Fasciola gigantica âgées de 12 jours : ce nombre est inférieur à celui (200 métacercaires) que recommandent les techniques modernes d'infestation, techniques qui ont été codifiées et standardisées par BORAY (1963, 1969). Il a été finalement choisi en tenant compte de la faible résistance des moutons locaux à Fasciola gigantica et du rendement Douves/Métacercaires (Au Tchad, en moyenne 30,2 p. 100).

Pour les jeunes zébus, le chiffre de 500 Métacercaires par tête a été retenu: c'est le plus favorable et le rendement Douves-Métacercaires est de 28,7 p. 100 environ (Rap. Farcha, 1969).

La seconde partie du protocole est demeurée très classique: elle a été exposée en détail à différentes reprises (GUILHON, GRABER et BIRGI, 1970). Il est donc inutile d'y revenir. Les deux médicaments ont été administrés « à la bouteille ", sans mise à la diète préalable. Le Bitin-S se présente sous l'aspect d'une poudre blanche renfermant 60 p. 100 de produit actif et l'Acédist sous la forme de bâtonnets de $4,5 \mathrm{~g}$ contenant $830 \mathrm{mg}$ de produit de base.

\section{RESULTATS}

Ils figurent aux tableaux 1, 2 et 3.

\section{Bitin-S}

\section{1. Chez le mouton (Tableau $\mathrm{n}^{\circ}$ 1)}

La dose de $30 \mathrm{mg} / \mathrm{kg}$ recommandée par GUILHON et GRABER (1966) n'est valable que pour les Trématodes mûrs présents dans les voies biliaires. Le traitement des distomatoses récentes - de 6 à 13 semaines exige une posologie légèrement plus élevée, de

(d) Dont deux agneaux infestés au Laboratoire de Parasitologie de 1'Ecole Nationale Vétérınaire de Lyon. l'ordre de $40 \mathrm{mg} / \mathrm{kg}\left({ }^{4}\right)$. Le pourcentage d'efficacité dépasse alors le seuil minimal de 90 p. 100 , tel qu'il a été défini par BORAY (1969).

\section{2. Chez le zébu (Tableau $\mathrm{n}^{\prime 2}$ )}

La dose de $30 \mathrm{mg} / \mathrm{kg}$ habituellement préconisée ( $\left.{ }^{5}\right)$ ne détruit qu'une partie des Distomes immatures de 75 jours. En deçà, elle est totalement inactive.

Entre la $8^{\mathrm{e}}$ et la $13^{\mathrm{e}}$ semaine faisant suite à l'infestation initiale, la dose de $40 \mathrm{mg} / \mathrm{kg}$, bien qu'irrégulièrement active, permet en moyenne l'élimination de plus de 90 p. 100 des parasites, ce qui est satisfaisant.

Pour les Trématodes âgés de 6 à 8 semaines, la posologie est comprise entre 40 et $60 \mathrm{mg} / \mathrm{kg}$. Déjà, à $40 \mathrm{mg} / \mathrm{kg}$, les mouvements des parasites placés dans de l'eau tiède sont fortement ralentis, surtout au niveau de leur région postérieure.

\section{3. Toxicité}

Le Bitin-S est capable de tuer l'ensemble des bouvillons mis en expérience vers $200 \mathrm{mg} / \mathrm{kg}$ et la totalité des moutons vers $250 \mathrm{mg} / \mathrm{kg}$. Cependant, dans cette espèce, des accidents toxiques mortels peuvent se produire dès $100 \mathrm{mg} / \mathrm{kg}$ (deux morts sur onze - GRABER, 1967). Cet état de choses limite donc singulièrement l'emploi du Disto-5 chez les ovins des zones tropicales $\left(^{6}\right)$ qui, à certaines époques de l'année, sont souvent en mauvais état d'entretien (fin de la saison sèche-début de l'hivernage, c'est-à-dire d'avril à août).

En revanche, chez le zébu, le coefficient de sécurité est bien meilleur : selon la dose administrée, il varie de 3,6 à 6,6 , ce qui, d'après les critères donnés par BORAY (1969), rend le médicament parfaitement utilisable dans l'espèce bovine, tant dans les pays chauds que dans les pays tempérés. C'est également l'opinion de GUILHON (1968).

\section{Acedist}

La lecture du Tableau $n^{0} 3$ montre que le Bromophénophos, à la dose de $10-12,5 \mathrm{mg} / \mathrm{kg}$

(4) Qui est celle indiquée par le fabricant.

(5) Fasciola gigantica adultes et mûres (14-16 semaines).

(6) Le mouton des zones tempérées semble moins sensible à l'anthelminthique. 
n'est efficace que sur les Fasciola de 103-104 jours presque mûres qui ont quitté le parenchyme hépatique et sont parvenues dans les canaux biliaires: 4 jours après le traitement, on retrouve dans la vésicule des parasites morts, diaphanes et de couleur verdâtre. Leur utérus renferme un grand nombre d'œufs.

Sur les formes immatures de 42 à 75 jours, l'Acédist n'a que peu d'effets, même lorsque l'on double la dose $(20-25 \mathrm{mg} / \mathrm{kg})$.

Par ailleurs, l'anthelminthique semble assez mal supporté par le zébu africain. Les doses de 50,75 et $100 \mathrm{mg} / \mathrm{kg}$ sont toutes mortelles. Les animaux intoxiqués présentent de violentes coliques, un état de prostration intense et une abondante diarrhée. A l'autopsie, on note une forte congestion intestinale accompagnée parfois d'hémorragies multiples.

Dans ces conditions, le médicament n'a que des indications très restreintes, c'est-à-dire traitement de la distomatose chronique de plus de 100 jours, à l'exclusion des distomatoses récentes en cours d'évolution, les doses thérapeutiques se rapprochant alors dangereusement des doses toxiques mortelles.

Ces résultats sont très différents de ceux obtenus dans l'espèce ovine: en effet, chez le mouton, quelle qu'en soit l'origine (EuropeAfrique), le Bromophénophos, à la dose de $22-25 \mathrm{mg} / \mathrm{kg}$, assure la destruction des Trématodes âgés de 6-10 semaines (GUILHON, GRABER et BARNABE, 1970; GUILHON, GRABER et BIRGI, 1970).

\section{CONCLUSIONS}

Les auteurs étudient le pouvoir antidistomien de deux médicaments récents sur les formes immatures de Fasciola gigantica durant leur migration dans le parenchyme hépatique.

Après avoir procédé à l'infestation expérimentale de moutons et de bouvillons neufs à l'aide de Métacercaires âgées de 12 jours, ils constatent:

1. Que l'Acédist, chez le zébu du Tchad, est peu actif sur les Distomes de moins de 14 semaines: la dose habituelle $(10-12,5 \mathrm{mg} / \mathrm{kg})$ qui assure la destruction dans les voies biliaires des parasites adultes de plus de 100 jours doit, en effet, être plus que doublée. Dans ces conditions, l'écart entre la dose thérapeutique et la dose toxique est trop faible et des accidents mortels risquent de se produire.

2. Que, chez le mouton, le Bitin-S, très efficace à $40 \mathrm{mg} / \mathrm{kg}$, doit être administré avec prudence, lorsque les animaux sont en mauvais état.

3. Que le même médicament, chez le zébu, est, en général, bien supporté: la marge de sécurité est largement suffisante. Les infestations de plus de 13 semaines exigent une dose de $30 \mathrm{mg} / \mathrm{kg}$, celles de 8 à 13 semaines $40 \mathrm{mg} / \mathrm{kg}$ avec un pourcentage d'efficacité de plus de 90 p. 100 et celles de moins de 8 semaines, une posologie comprise entre 40 et $60 \mathrm{mg} / \mathrm{kg}$.

\title{
SUMMARY
}

\section{Efficiency of Bitin-S and Bromophenophos on immature Fasciola gigantica}

\begin{abstract}
In tropical countries, the treatment of recent fascioliasis (less than fourteen weeks) is difficult.

Acédist, in zebu cattle, is unserviceable, because the usual dose $(10-12,5 \mathrm{mg} / \mathrm{kg})$ must be more than doubled and, so, the drug might be toxic at neighbouring doses.

Bitin-S, which is very efficace in sheep (about $40 \mathrm{mg} / \mathrm{kg}$ ) should not be administered to flocks in too poor condition. But, in zebu, this medicament is generally well tolerated. For eight to thirteen weeks old flukes, the best dose is $40 \mathrm{mg} / \mathrm{kg}$, with an efficiency more than $90 \mathrm{p} .100$. Underneath, it must be lightly increased.
\end{abstract}

\section{RESUMEN}

A propósito de la acción del Bitin $\mathbf{S}$ y del Bromophenophos sobre Ias formas immaduras de Fasciola gigantica

En región tropical, es dificil el tratamiento de las distomatosis 
recientes causadas por formas inmaduras de Fasciola gigantica en migración en el parénquima hepático.

En el cebú, Acedist es inutilizable, por que se necesita más que doblar la dosis habitual $(10-12,5 \mathrm{mg} / \mathrm{kg}) \mathrm{y}$, en estas condiciones, el medicamento puede hacerse peligroso para la salud del animal.

En la oveja, no se tendra que administrar el Bitin $\mathrm{S}$, aunque sea muy eficaz una dosis de unos $40 \mathrm{mg} / \mathrm{kg}$, en ovejas en demasiado malo estado.

En cambio, el cebú generalmente tolera bien el medicamento.

Es de $40 \mathrm{mg} / \mathrm{kg}$ la dosis preconizada para infestaciones de 8 a 13 semanas, con un porcentaje de eficacidad superior a 90 p. 100. Las de menos de 8 semanas exigen una posologia un poco más elevada.

\section{BIBLIOGRAPHIE}

BORAY (J.C.), « Standardization of techniques for pathological and anthelmintic studies with Fasciola spp \#, Proc. Symp. Evaluation of anthelmintics, Hanovre, 1963. Merck Sharp and Dohme, 1964, pp. 43-45.

BORAY (J.C.), « Experimental fascioliasis in Australia », $A d v$. Parasit., 1969, 7 : 95-210.

CHROUSTOVA (E.), WILLOMITZER (J.), BOROVANSKY (A.), « The testing of bis ( 2 hydroxy, 3,5-Dichlorophenyl of Sulphoxide BH-S against fascioliasis of cattle ", Veternaria Prague, 1969, 11 (4) : 287-302.

DELAK (M.), MAGUD (I.), « Komparative prufung der Fascioloziden wirkung yon Zanil, Bitin-S und Bayer 91015 beim Rind ", Wad. Parcayt., 1968, 14 (5-6): 659-66.

EUZERY (I.), «Données modernes concernant le traitement et la prophylaxie des helminthoses digestives des bovins ", Rev. Med. Vet., 1968, 119 (5) : 475-516.

GRABER (M.), Rapp. a. Lab. Farcha, 1967, 3: 128.

GUILHON (J.), GRABER (M.), "Action du bis (2-Hydroxy-3,5, dichlorophényl) Sulfoxyde sur divers Trématodes parasites des ruminants", Bull. Acad. vét. Fr., 1966, 39 (9) : 307-12.

GUILHON (J.), GRABER (M.), «Etude en milieu tropical du pouvoir anthelminthique du Bis (2-Hydroxy 3,5-Dichlorophényl) Sulfoxyde », Rev. Elev. Méd. vét. Pays trop., 1967, 20 (1) 87-104.

GUILHON (J.) "Chimiothérapie spécifique de la fasciolose hépatique », Wiad. Parazyt., 1968, 14 (5-6) : 589-93.

GUILHON (J.), GRABER (M.), BARNABE (R.), «Action fasciolicide d'un nouvel ester phosphorique tetrabromé et sa toxicité pour le mouton ", Bull. Acad. vét. Fr., 197043 (2): 67-73.

GUILHON (J.), GRABER (M.), BIRGI (E), « Etude du pouvoir anthelminthique du Bromophénophos à l'égard de divers endoparasites du mouton et du zébu de la République du Tchad », Rev. Elev. Méd. vét. Pays trop., 1970, 23 (2): 199-206.

KOOPMAN (J.J.), a Field trials of anthelmintics against bovine fascioliasis. Report of the Dutch committee of animal health (on Hexachlorophène, Menichlopholan and Acédist)", Tijdschr. Diergeneesk., 1969, 94, 1393-1408.

KRUYT (W.), VAN DER STEEN (E.J.), « Experiments with a new anthelmuntic against the liverfluke », Tijdschr. Diergeneesk., 1969, 94, 308-23.

ODA (A.), "Treatment of Liver-fluke infection in cattle with A.K.T. 501 (Bitin-S)», Nakakambara Livestock Hyg. Serv. Cent., 1962.

Rapp. a. Lab. Farcha, 1969. Pp. 256-78.

REINDEERS (J.S.), «Field trials in cows of a new drug against Distomatosis (PH 1882): comparison with Hexachlorophène and testing for side effects ", Tijdschr. Diergeneesk., 1969, 94, 324-30.

UENO (H.), WATANABE (S.). FUJITA (J.), " Anthe!mintic activity of chlorinated Diphenyl Sulfides and related compounds of the Liver-fluke, Fasciola gigantica in experimentally infected rabbits $"$ Nat. Inst. Anim. Hith. Quart. Tokyo, 1964, 4 (2) : 77-85.

UENO (H.), WATANABE (S.), FUJITA (J.), « Comparison of the anthelmintic effects of Chlorinated Diphenyl Sulfides and their Methane Derivative in the Liver-fluke Fasciola grgantica on rabbits and sheep", Nat. Inst Anim. Hith. Quart. Tokyo, 1964, 4 (3): 168.66.

VAN DER MEER (S.), POUWELS (H.), " 4,4 ',6,6" tetrabromo-2,2'-Biphenycdiol mono (Dihydrogen phosphate), a new agent for combating distomatosis », J. Med. Chem., 1969, 12, 534. 